Received: 9 February 2018

Accepted: 5 October 2018

Published online: 15 October 2018

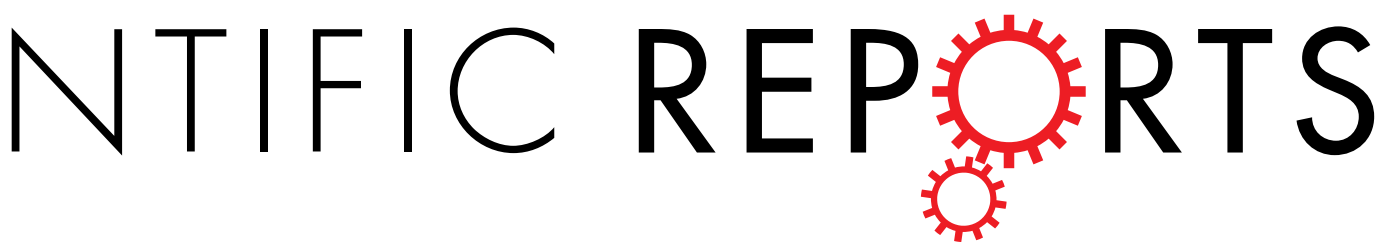

\title{
OPEN A facile and general approach for production of nanoscrolls with high-yield from two-dimensional nanosheets
}

\author{
Wucong Wang, Yanzhe Gai, Ding Xiao \& Yaping Zhao
}

Nanoscrolls (NSs) assembled from two-dimensional nanosheets have emerged as a novel type of one-dimensional nanomaterials because of their unique topological features and properties. The scale-up preparation of the NSs is crucial for their foundational and applied research. Herein, we report a general and straightforward approach for efficiently converting two-dimensional nanosheets into the NSs with high yield. We demonstrated the converting process by illustrating the formation of the graphene nanoscrolls through characterizing their morphology and structure using a scanning electron microscope, transmission electron microscope, Raman spectra, and X-ray diffraction spectra. The graphene sheets with a few-lay number were converted immediately and entirely into the graphene nanoscrolls when they mixed with an ethanol solution of silver nitrate at room temperature. The as-prepared graphene nanoscrolls were confirmed to be formed via the layer-by-layer assembly of graphene triggered by silver cyanide formed in site. Also, we extended this approach to construct the nanoscrolls of the hexagonal boron nitride, molybdenum disulfide, and tungsten disulfide, respectively, from their corresponding two-dimensional nanomaterials. In a broader context, this approach paves a significant new way for the large production of the NSs with cost-efficiency.

Carbon nanoscrolls were discovered when making multiwalled carbon nanotubes ${ }^{1,2}$. Since the discovery of graphene, the carbon nanoscrolls was convinced of deriving from scrolling of graphene, named as graphene nanoscrolls (denoted GNSs) ${ }^{3,4}$. GNSs inherit not only excellent properties of graphene, such as high mechanical strength, outstanding electrical conductivity, and superior carrier mobility but also possess open topological structure at both ends and interlayer galleries ${ }^{5}$. It endows the GNSs with wonderful features, such as excellent electronic and electromechanical properties ${ }^{6,7}$. Thus, the GNSs have significant potential applications in energy storages, photovoltaic cells and sensors ${ }^{4,8-10}$. Besides graphene, recently it was reported that the other layered two-dimensional nanomaterials (denoted 2DNMs) could be rolled into the nanoscrolls (denoted NSs) too, such as h-boron nitride (h-BN), tungsten disulfide $\left(\mathrm{WS}_{2}\right)$, and molybdenum disulfide $\left(\mathrm{MoS}_{2}\right)^{11}$. The NSs have become a new type of one-dimension nanomaterials with novel properties. Therefore, the preparation of the NSs has attracted increasing attention of scientific researchers. However, most of the research work is limited to theoretical prediction and calculation ${ }^{12-15}$. Only a few previously published articles involved fabricating the NSs. Moreover, the reported processes are complicated, and the conversion yield is tiny limiting their application ${ }^{16-23}$. Up to date, there is no general and efficient method to enable assembling the NSs with high-yield.

Herein, we report a facile and general approach for production of high-quality NSs with high-yield from two-dimensional nanosheets. The NSs are fabricated via self-scrolling of the 2DNMs in the solution of silver nitrate and ethanol. We have demonstrated the formation of the nanoscrolls of graphene, h- $\mathrm{BN}, \mathrm{MoS}_{2}$, and $\mathrm{WS}_{2}$ by verifying the morphology and structure of the formed NSs using scanning electron microscope, transmission electron microscope, Raman spectra, and X-ray diffraction spectra. Also, the mechanism of the self-assembly scrolling processes is elucidated. 

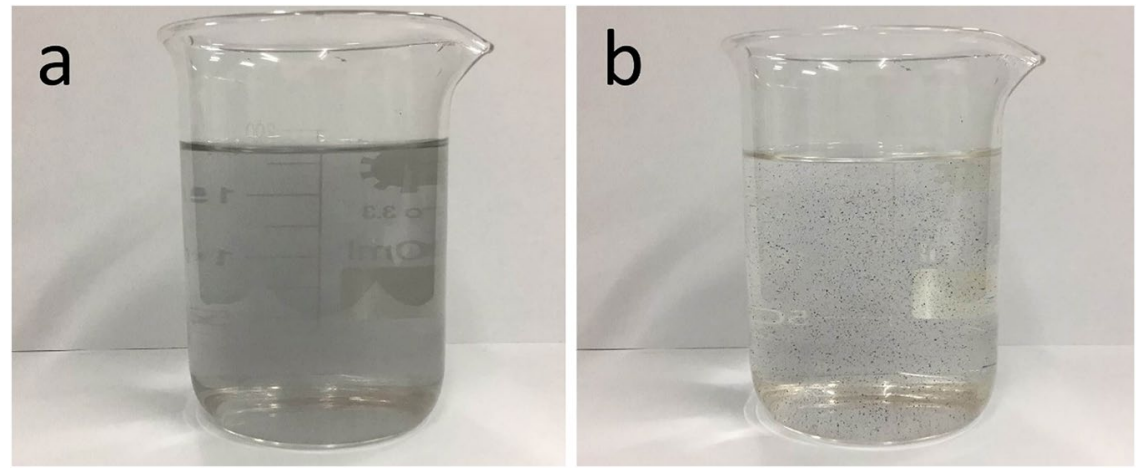

Figure 1. Digital photograph of (a) the dispersion of graphene in ethanol, and (b) the graphene nanoscrolls formed in ethanol solution.
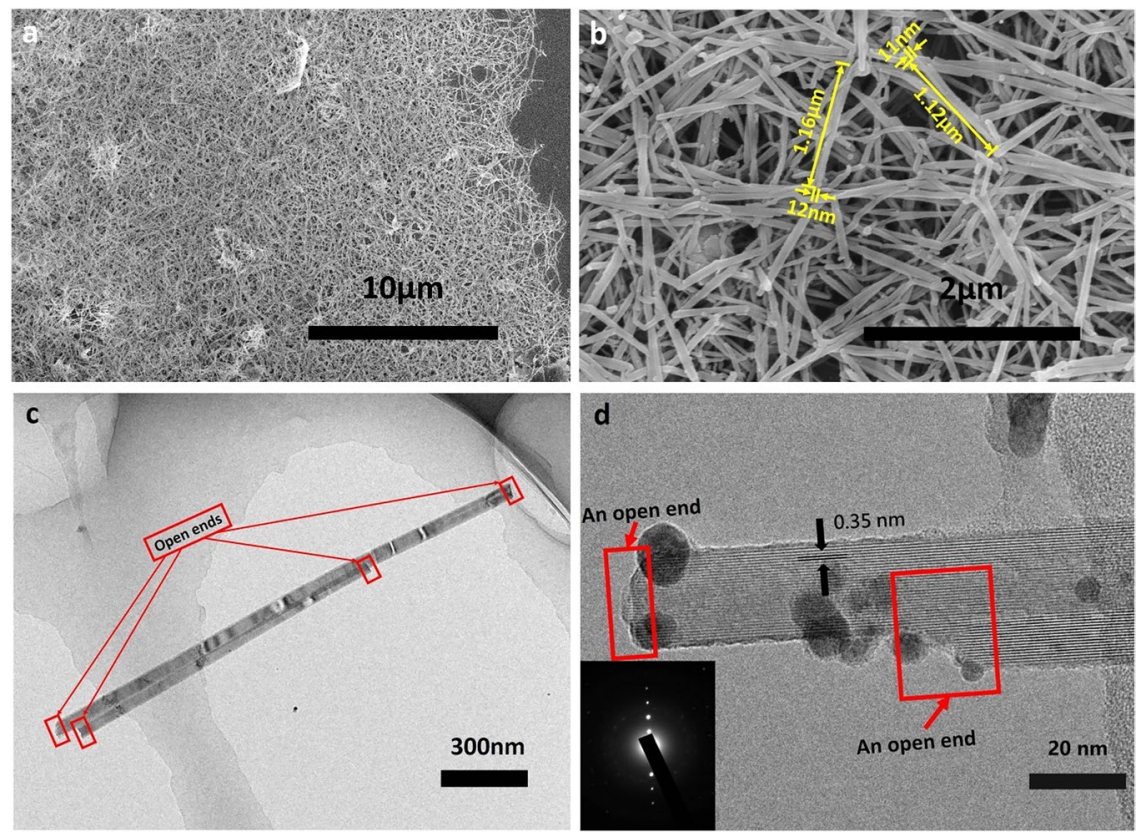

Figure 2. Microstructure and morphology of the GNSs. (a,b) SEM images of GNSs under different magnifications. (c) TEM image of two abreast GNSs. (d) High-magnification TEM image of the GNS and its typical electron diffraction pattern.

\section{Results and Discussion}

The graphene sheets exfoliated from graphite are taken as the representative of the 2DNMs to discuss in detail. The profiles of the exfoliated graphene sheets are shown in Fig. S1, which indicates that the graphene is less than five layers with the lateral size of 1-3 $\mu \mathrm{m}$. The appearance change of the reaction solution during the conversion of the graphene to the GNSs was recorded by a digital camera. Figure 1a displayed the initial state of the grey dispersion of the graphene and $\mathrm{AgNO}_{3}$ ethanol solution when they were mixed and magnetically stirred. After stirring the solution for some time, the grey dispersion gradually changed into a clear solution (Fig. 1b), from which it can be seen that a kind of fluffy particles was formed and deposited. These particles are confirmed to be the GNSs by a series of characterization methods.

A scanning electron microscope (SEM) and a transmission electron microscope (TEM) were applied to characterize the microstructure and morphology of the GNSs. The SEM image (Fig. 2a) indicates that a mass of the GNSs was formed and the graphene sheets disappeared. It means that all of the exfoliated graphene sheets have been converted into the GNSs. It can be seen from the high-magnification SEM (Fig. 2b) that the GNSs uniformly scattered on the substrate and the length and diameter of the GNSs are in the range of $0.5-10 \mu \mathrm{m}$ and $10-50 \mathrm{~nm}$, respectively, which relates to the size of the original graphene sheets and the curling mode. The TEM images illustrate further the microstructure of the GNSs. Two abreast GNSs are shown in Fig. 2c. Their ends are open, and their lengths and diameters are $1.2-1.8 \mu \mathrm{m}$ and $20-30 \mathrm{~nm}$, respectively. The sizes of the GNSs measured from the TEM are consistent with ones from the SEM. The high-magnification TEM (Fig. 2d) displays that the interlayer spacing of the GNSs is about $0.35 \mathrm{~nm}$ which is as similar as the (002) distance of graphite. It suggests that the 
a

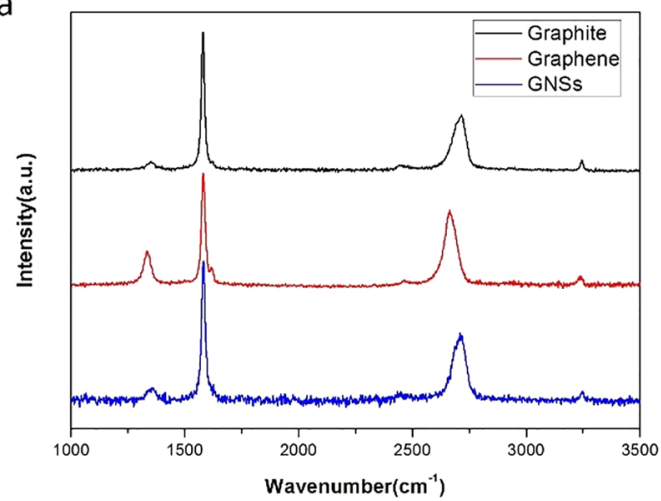

$\mathrm{b}$

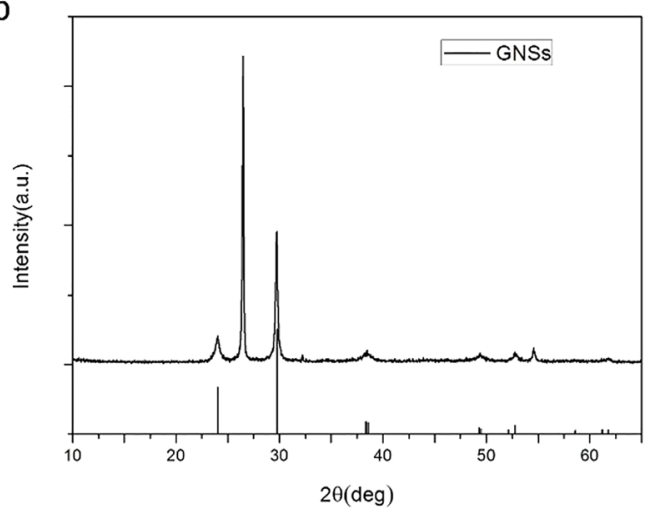

Figure 3. Raman and XRD characterization. (a) Raman spectrum of graphite, graphene and GNSs, (b) XRD patterns of GNSs and PDF card of AgCN (PDF\#23-1404).
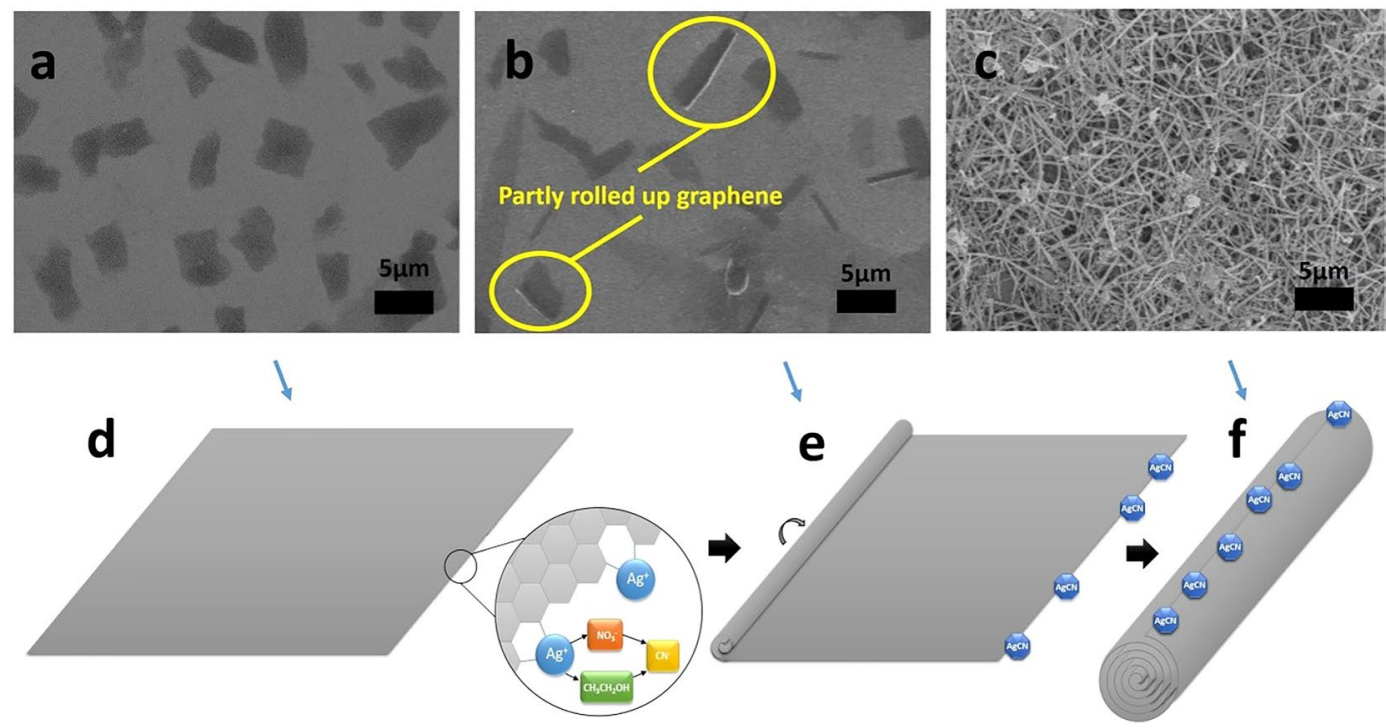

Figure 4. SEM images of the graphene (a), intermediate transition state (b) and the converted GNSs (c). The speculation mechanism of the scrolling process of the graphene into the GNSs. A square graphene sheet and the position occupied with the created $\mathrm{AgCN}(\mathbf{d})$, the partially curled state of the graphene and the formed $\mathrm{AgCN}$ particles at the edge (e), the final finished GNSs and formed AgCN particles on the surface of the GNSs (f).

GNSs were scrolled up via layer by layer. Also, the typical electron diffraction pattern (inset of Fig. 2d) indicates the specific structure of the GNSs has an identical structure to the multiwalled carbon nanotubes ${ }^{24}$. However, the ends of the GNSs are open not like the closed structure of the multiwalled carbon nanotubes as shown in the red rectangles of Fig. 2 d.

Raman spectrum is an excellent way to characterize the structure of the nanostructured materials. Comparing the Raman spectrum of the graphite, the graphene, and the GNSs shown in Fig. 3a, we can see that the structure of the GNSs is more similar to the bulk graphite rather than to the graphene sheets except that their frequency and intensity of the characteristic peaks are a little different. The ratio of G peak $(\sim 1580 \mathrm{~cm}-1)$ to $2 \mathrm{D}$ peak $(\sim 2700$ $\mathrm{cm}^{-1}$ ) and the shift of the 2D peak are often used to judge the layer number ${ }^{22}$. Accordingly, the graphene sheets which scrolled to the GNSs are a few layer numbers which are agreement with the results characterized by TEM and AFM shown in Fig. S1. The interlayer spacing of the GNSs is also calculated to be around $0.34 \mathrm{~nm}$ according to the value of $2 \theta$ of the (002) peaks of the XRD patterns of the GNSs (Fig. 3b), which matches well with the TEM and Raman spectrum. Moreover, we have proved that the nanoparticles on the surface of the GNSs shown in Fig. $2 \mathrm{~d}$ are AgCN by comparing the diffraction characteristic peaks of the GNS with the PDF card of AgCN as shown in Fig. 3b. The detail demonstration work has been reported in the recently published article ${ }^{25}$.

In order to explore the conversion process of the graphene to the GNSs, we have examined the change of the morphology and structure of the graphene with the reaction period via the SEM characterization. Figure $4 a-c$ illustrates the morphology of the initially exfoliated graphene, the partially-curling graphene, and the GNSs. The profile of the typical exfoliated-graphene sheets before the reaction is displayed in Fig. 4a. It can be seen that most of the layers are rectangle-like and uniform in size. When the reaction went in $10 \mathrm{~min}$, we can see from 


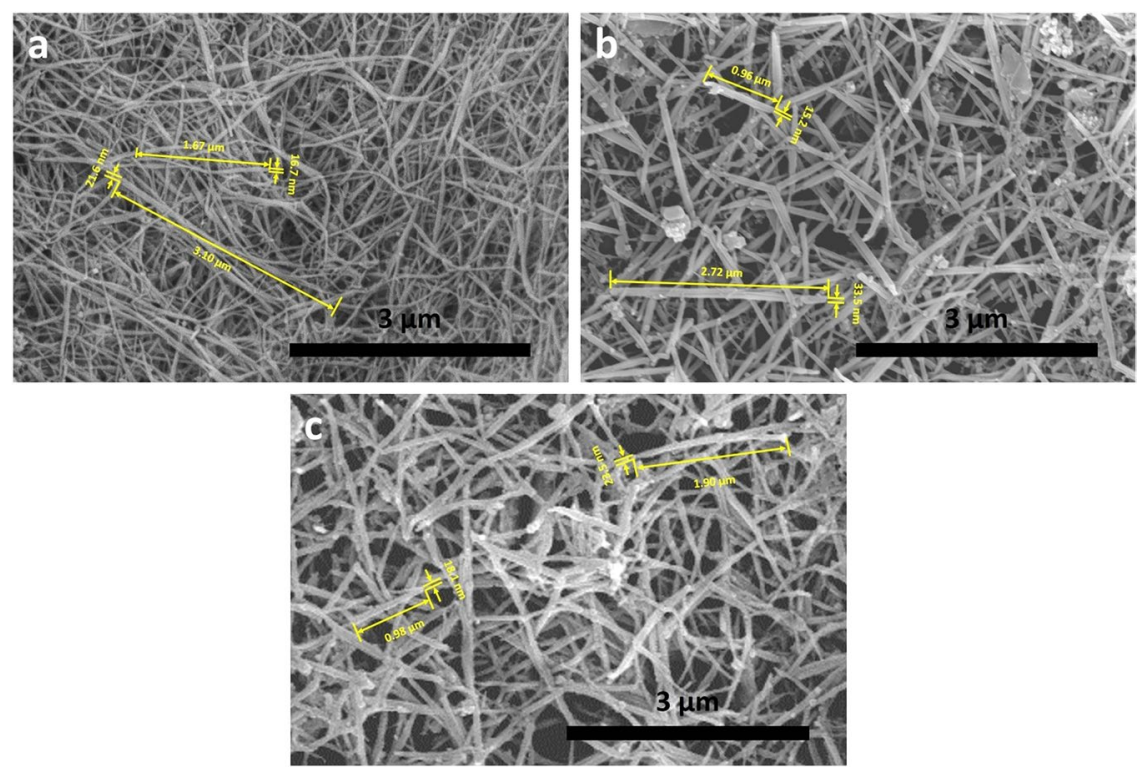

Figure 5. Micrograph of the NSs. SEM images of (a) the NSs of h-BN, (b) the NSs of $\mathrm{MoS}_{2}$ and (c) the NSs of $\mathrm{WS}_{2}$.

Fig. $4 \mathrm{~b}$ that the graphene sheets had been partly rolled up. When the reaction continued to go on for $30 \mathrm{~min}$, all the graphene sheets had been scrolled up into the GNSs (Fig. 4c). The curling process of the graphene into the GNSs can be explained in the way as shown in the Fig. $4 \mathrm{~d}-\mathrm{f}$. The AgCN nanoparticles generated at the edge of the graphene play a crucial role in the formation of the GNSs. Initially, the AgCN was created at the edge of the graphene because of the activated dangling bonds of the graphene when the ethanol solution of the graphene was mixed with the ethanol solution of the silver nitrate. The resulted $\mathrm{AgCN}$ would change the electron density on the surface of the graphene affecting the adsorption of ethanol solvent on the graphene, increasing the surface energy of the graphene. Thus, when the surface energy of the graphene accumulated to a high level with the increase of the forming AgCN particles (Fig. 4e), the few-layer graphene sheets were triggered to curl up to reduce its surface energy until the complete-scrolls (GNSs) were formed (Fig. 4f). The newly generated AgCN particles trigger the graphene sheets to scroll up. Once the edge of the end of the graphene sheet overlaps its layer, the scrolling-up process continues to the end because of the van der Waals interactions. At this time, the bending energy of the graphene is offset by the der Waals interactions. This speculation is proved by the position of the AgCN particles on the GNSs, all of which are found to be at the exposed edge of the GNSs (Figs 2d and S2). Also, the speculation can be proved by comparing the real size of the GNSs measured from the SEM and TEM images with that calculated based on the mechanism. Suppose a graphene sheet is a square shape and the rolling way is layer-by-layer self-assembly (Fig. S3), the diameter of the GNSs can be calculated to be about $15 \mathrm{~nm}$ and $66 \mathrm{~nm}$ based on the length of the GNSs ranging from $0.5 \mu \mathrm{m}$ to $10 \mu \mathrm{m}$. The calculation results (the relationship between the length and the diameter of the GNSs) are in good agreement with that made from the SEM and TEM images (Fig. 2a-d). It suggests that the mechanism of the forming GNSs aforementioned is reasonable. It is worth to mention that the GNSs should have superior electronic property because the electron conduction inside the GNSs is on the same graphene surface.

We extend this approach to make other 2DNMs such as h-BN, $\mathrm{MoS}_{2}$, and $\mathrm{WS}_{2}$ to explore its generality. The SEM images (Fig. 5a-c) indicated that the exfoliated 2DNMs had been converted into the nanoscrolls (denoted NSs) with a high yield. The Raman spectrum of the bulk h-BN, the bulk $\mathrm{MoS}_{2}$, the bulk $\mathrm{WS}_{2}$, their initially exfoliated nanosheets, and the NSs indicate that these bulk 2DNMs were peeled off into a few-layer number and in turn converted into the NSs (see Section 4 in supplementary materials). In the similar way of forming the GNSs, the formed $\mathrm{AgCN}$ triggered the 2DNMs to transform into the NSs. The AgCN nanoparticles on the NSs were confirmed from the XRD patterns of the NSs of the h-BN, $\mathrm{MoS}_{2}$, and $\mathrm{WS}_{2}$ (see Section 4 in supplementary materials). Also, we investigated the influence of the mass ratio of $2 \mathrm{DNMs}$ and $\mathrm{AgNO}_{3}$ on the fabrication of the NSs. As shown in Fig. S5(b,c), only when the mass-ratio between $\mathrm{AgNO}_{3}$ and 2DNMs is 0.002 or more, can the NSs be formed. The diameter of the NSs made from the h-BN was calculated to be from $15 \mathrm{~nm}$ to $65 \mathrm{~nm}$ based on the length varying from $0.5 \mu \mathrm{m}$ to $10 \mu \mathrm{m}$, respectively, which are accorded with the result made via SEM (Fig. 5). The ratio between the length and the diameter is almost as the same as the GNSs. It might be attributed to the fact the $\mathrm{h}-\mathrm{BN}$ has the same interlayer space as the graphite. Similarly, the relationship between the calculated diameter and the length of the NSs made from the $\mathrm{MoS}_{2}$ and $\mathrm{WS}_{2}$ are consistent with the results obtained from the SEM. Therefore, these results support the scrolling mechanism aforementioned, and the scrolling process reported in this work is universal.

In conclusion, we have demonstrated a simple and general method to produce high-quality NSs. The single- or few-layer 2DNMs can quickly curl up into the NSs by mixing their ethanol solution with $\mathrm{AgNO}_{3}$ ethanol solution at room temperature. It is the $\mathrm{AgCN}$ particles generated at the edge of the $2 \mathrm{DNMs}$ sheets that trigger the curling 
process. The successful formation of the NSs from the exfoliated Graphene, h-BN, $\mathrm{MoS}_{2}$, and $\mathrm{WS}_{2}$, respectively, substantiates that this approach can be potentially extended to other 2DNMs. Also, the conversion of 2DNMs into their corresponding NSs reaches almost $100 \%$. This work paves the way for largely producing the NSs and carrying out the foundational study and practical application of the NSs.

\section{Methods}

Chemicals and Reagents. Graphite powder was purchased from Sinopharm Chemical Reagent Co., Ltd (China). H-BN bulk powder was obtained from Strem Chemicals. The $\mathrm{MoS}_{2}$ bulk powder was purchased from Alfa Aesar. The $\mathrm{WS}_{2}$ bulk powder was purchased from Aladdin. Ethanol (99.7\%), $\mathrm{AgNO}_{3}$ were purchased from Sinopharm Chemical Reagent Co., Ltd (China). Carbon dioxide (99.9\%) was obtained from Shanghai high-tech Co., Ltd (China).

Exfoliation of 2DNMs. Graphene, h-BN, $\mathrm{MoS}_{2}$, and $\mathrm{WS}_{2}$ were exfoliated using a shear mixer in a supercritical $\mathrm{CO}_{2}$ approach similar to the previously reported paper $^{26}$. The $1 \mathrm{~g}$ raw material was put into the reactor, and carbon dioxide was pumped into the reactor by the manual pump. When the pressure and the temperature reached a preset value of $12 \mathrm{MPa}$ and $45^{\circ} \mathrm{C}$, the shear mixer was started and remained at a speed of $3000 \mathrm{r} / \mathrm{min}$ for 1 hour. The resultant sample was dispersed by an ultrasonic water bath and centrifuged. More than $80 \%$ of the exfoliated 2DNMs were confirmed to be 1 to 5 layers by AFM, TEM and Raman spectra.

Synthesis of NSs. A certain amount of the exfoliated 2DNMs and $\mathrm{AgNO}_{3}$ was dispersed in ethanol solvent in a predefined mass ratio. When the dispersion was magnetically stirred for $30 \mathrm{~min}$ at room temperature, the 2DNMs rolled up and deposited as shown in Figs 1 and S6. Then the resulted sediment was separated and dried at $60^{\circ} \mathrm{C}$ to obtain the NSs. The as-prepared NSs were stored in the bottle for later characterization.

Characterization. Scanning electrode microscope imaging was performed using an FEI Nova NanoSEM 450 (USA). Transmission electron microscope images were obtained using JEOL JEM-2100 TEM (Japan) at an acceleration voltage of $120 \mathrm{kV}$. The X-ray diffraction (XRD) recorded on a BRUKER D8 Advance X-Ray Diffractometer (Germany) using a Cu K $\alpha$ radiation source $(\lambda=1.5418 \AA)$. The Raman spectroscopy was recorded on a Raman Microscope excitation (USA) with a wavelength of $532 \mathrm{~nm}$. Zeta Potential was performed with a Malvern Instruments ZS90 Particle Size and Zeta Potential Analyzer (UK).

\section{References}

1. Amelinckx, S., Bernaerts, D., Zhang, X. B., Tendeloo, G. V. \& Landuyt, J. V. A Structure Model and Growth Mechanism for Multishell Carbon Nanotubes. Science 267, 1334 (1995).

2. Ruland, W., Schaper, A. K., Hou, H. \& Greiner, A. Multi-wall carbon nanotubes with uniform chirality: evidence for scroll structures. Carbon 41, 423-427 (2003).

3. Viculis, L. M., Mack, J. J. \& Kaner, R. B. A chemical route to carbon nanoscrolls. Science 299, 1361 (2003).

4. Xie, X. et al. Controlled fabrication of high-quality carbon nanoscrolls from monolayer graphene. Nano Letters $\mathbf{9}, 2565$ (2009).

5. Braga, S. F. et al. Structure and Dynamics of Carbon Nanoscrolls. Nano Letters 4, 881-884 (2015).

6. Rurali, R., Coluci, V. R. \& Galv Atilde, D. S. Prediction of giant electroactuation for papyruslike carbon nanoscroll structures: Firstprinciples calculations. Physical Review B 74 (2006).

7. Schaper, A. K., Wang, M. S., Xu, Z., Bando, Y. \& Golberg, D. Comparative studies on the electrical and mechanical behavior of catalytically grown multiwalled carbon nanotubes and scrolled graphene. Nano Letters 11, 3295-3300 (2012).

8. Sharifi, T. et al. Formation of nitrogen-doped graphene nanoscrolls by adsorption of magnetic $\gamma$-Fe2O3 nanoparticles. Nature Communications 4, 2319 (2013).

9. Yan, M. et al. Nanowire templated semihollow bicontinuous graphene scrolls: designed construction, mechanism, and enhanced energy storage performance. Journal of the American Chemical Society 135, 18176 (2013).

10. Zheng, B., Xu, Z. \& Gao, C. Mass production of graphene nanoscrolls and their application in high rate performance supercapacitors. Nanoscale 8, 1413 (2016).

11. Lai, Z., Chen, Y., Tan, C., Zhang, X. \& Zhang, H. Self-Assembly of Two-Dimensional Nanosheets into One-Dimensional Nanostructures. Chem 1, 59-77 (2016).

12. Hwang, D. Y., Choi, K. H., Park, J. E. \& Suh, D. H. Evolution of magnetism by rolling up hexagonal boron nitride nanosheets tailored with superparamagnetic nanoparticles. Physical Chemistry Chemical Physics Pccp 19, 4048 (2017).

13. Hwang, D. Y. \& Suh, D. H. Evolution of a high local strain in rolling up MoS2 sheets decorated with Ag and Au nanoparticles for surface-enhanced Raman scattering. Nanotechnology 28, 025603 (2017).

14. Perim, E. \& Galvao, D. S. Stability and Dynamics of Boron Nitride Nanoscrolls. Nanotechnology 20, 335702 (2009).

15. Perim, E., Paupitz, R. \& Galvao, D. S. Controlled route to the fabrication of carbon and boron nitride nanoscrolls: A molecular dynamics investigation. Journal of Applied Physics 113, 283 (2013).

16. Chen, X., Boulos, R. A., Dobson, J. F. \& Raston, C. L. Shear induced formation of carbon and boron nitride nano-scrolls. Nanoscale 5, 498-502 (2013).

17. Dresselhaus, M. S., Jorio, A., Hofmann, M., Dresselhaus, G. \& Saito, R. Perspectives on carbon nanotubes and graphene Raman spectroscopy. Nano Letters 10, 751 (2010).

18. Hwang, D. Y. \& Suh, D. H. Formation of hexagonal boron nitride nanoscrolls induced by inclusion and exclusion of self-assembling molecules in solution process. Nanoscale 6, 5686-5690 (2014).

19. Meng, J. et al. Rolling Up a Monolayer MoS2 Sheet. Small 12, 3770-3774 (2016).

20. Tan, C. et al. Self-assembled chiral nanofibers from ultrathin low-dimensional nanomaterials. Journal of the American Chemical Society 137, 1565 (2015).

21. Thangasamy, P. \& Sathish, M. Rapid, one-pot synthesis of luminescent MoS2 nanoscrolls using supercritical fluid processing. Journal of Materials Chemistry C 4, 1165-1169 (2016).

22. Zheng, J. et al. Production of high-quality carbon nanoscrolls with microwave spark assistance in liquid nitrogen. Advanced Materials 23, 2460-2463 (2011).

23. Hwang, D. Y., Choi, K. H., Park, J. E. \& Suh, D. H. Highly thermal-stable paramagnetism by rolling up MoS2 nanosheets. Nanoscale 9, 503 (2016).

24. Andrews, R., Jacques, D., Qian, D. \& Dickey, E. C. Purification and structural annealing of multiwalled carbon nanotubes at graphitization temperatures. Carbon 39, 1681-1687 (2001). 
25. Xiao, D., Wang, W., Gai, Y. Z. \& Zhao, Y. P. Graphene-catalyzed formation of $\mathrm{C} \equiv \mathrm{N}$ bonds via cleavage of C-C and N-O bonds in ethanol and nitrate under room temperature. Scientific REPORTs 8, 1750, https://doi.org/10.1038/s41598-018-20238-y (2018).

26. Song, N. et al. Green production of pristine graphene using fluid dynamic force in supercritical CO2. Chemical Engineering Journal 298, 198-205 (2016).

\section{Acknowledgements}

We acknowledge funding from the National Natural Science Foundation of China (Grants No. 21576165), and Instrumental Analysis Center of SJTU for analysis.

\section{Author Contributions}

W.W. and Y.Z. conceived and designed the experiments. W.W. carried out NSs synthesis. W.W. and Y.G. performed characterization. W.W., Y.G. and D.X. analyzed the data. W.W. and Y.Z. wrote the paper. All authors discussed the results and commented on the manuscript.

\section{Additional Information}

Supplementary information accompanies this paper at https://doi.org/10.1038/s41598-018-33709-z.

Competing Interests: The authors declare no competing interests.

Publisher's note: Springer Nature remains neutral with regard to jurisdictional claims in published maps and institutional affiliations.

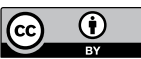

Open Access This article is licensed under a Creative Commons Attribution 4.0 International format, as long as you give appropriate credit to the original author(s) and the source, provide a link to the Creative Commons license, and indicate if changes were made. The images or other third party material in this article are included in the article's Creative Commons license, unless indicated otherwise in a credit line to the material. If material is not included in the article's Creative Commons license and your intended use is not permitted by statutory regulation or exceeds the permitted use, you will need to obtain permission directly from the copyright holder. To view a copy of this license, visit http://creativecommons.org/licenses/by/4.0/.

(C) The Author(s) 2018 